\title{
Adults with High-functioning Autism Process Web Pages With Similar Accuracy but Higher Cognitive Effort Compared to Controls
}

\author{
Victoria Yaneva* \\ v.yaneva@wlv.ac.uk \\ Sukru Eraslan** \\ seraslan@metu.edu.tr
}

\author{
Le An Ha* \\ Ha.L.A@wlv.ac.uk \\ Yeliz Yesilada** \\ yyeliz@metu.edu.tr
}

\begin{abstract}
${ }^{*}$ Research Institute in Information and Language Processing, University of Wolverhampton, UK ${ }^{* *}$ Middle East Technical University, Northern Cyprus Campus, Kalkanli, Guzelyurt, Mersin 10, Turkey
\end{abstract}

\begin{abstract}
To accommodate the needs of web users with high-functioning autism, a designer's only option at present is to rely on guidelines that: i) have not been empirically evaluated and ii) do not account for the different levels of autism severity. Before designing effective interventions, we need to obtain an empirical understanding of the aspects that specific user groups need support with. This has not yet been done for web users at the high ends of the autism spectrum, as often they appear to execute tasks effortlessly, without facing barriers related to their neurodiverse processing style. This paper investigates the accuracy and efficiency with which high-functioning web users with autism and a control group of neurotypical participants obtain information from web pages. Measures include answer correctness and a number of eye-tracking features. The results indicate similar levels of accuracy for the two groups at the expense of efficiency for the autism group, showing that the autism group invests more cognitive effort in order to achieve the same results as their neurotypical counterparts.
\end{abstract}

\section{CCS Concepts}

-Human-centered computing $\rightarrow$ User studies; Webbased interaction; Empirical studies in accessibility; Accessibility design and evaluation methods;

\section{Keywords}

Autism, Web Accessibility, Eye Tracking, Inclusive Design

\section{INTRODUCTION}

Autism Spectrum Disorder (ASD) is a neurodevelopmental disorder that primarily affects communication and social interaction [1], as well as other areas such as attention shifting, reading comprehension, and the transfer of information from one domain to another [9]. The autism diagnosis includes a spectrum of abilities and needs, where people at the high end of the spectrum are highly independent and people at the lower ends often rely on a caregiver.

Within the web accessibility community there has not yet been an in-depth discussion, nor empirical research, on the implications that different levels of autism severity have on the requirements for web accessibility. Web users on the higher ends of the spectrum often appear not to struggle compared to users at the lower ends of the spectrum, while there is no evidence to suggest that this is indeed the case. This assumption relates to the fact that many of these web users have a high level of independence and are able to complete tasks equally competently compared to their neurotypical counterparts. Nevertheless, whether or not their noeurodiversity has an impact on the way they cope with specific information processing tasks remains an open question.

In this paper, we investigate the accuracy and efficiency with which web users with autism and control group participants obtain information from web pages. Accuracy relates to the ability of the participants to obtain a specific piece of information through examining the page elements and is measured through their responses to information searching questions as being correct or incorrect. Efficiency is related to the speed and cognitive effort with which the relevant elements are located and the necessary information is extracted. It is measured through the time it takes to answer the questions, as well as eye-tracking measures. Our focus is particularly on highly able people on the autism spectrum, including those living independently without relying on a caregiver, people who are in full-time employment, and/or people currently enrolled in a higher education degree. We collect data from 19 people with autism and 19 control group participants using eight web pages with varying levels of complexity and distance between elements. Our full set of stimuli are available at: https://tinyurl.com/y9olxpu6.

\section{AUTISM AND WEB ACCESSIBILITY}

People with autism often exhibit idiosyncratic visual attention patterns. This is evidenced by eye tracking studies, where they are reported to focus on smaller bits of infor- 
mation to the exclusion of the bigger picture [10]. Such differences in attention shifting, when present, are found throughout the entire spectrum.

While scarce, there is also evidence suggesting that atypical visual attention causes web users with autism to interact with the web differently. Previous work reports that "people with autism tend to employ different information searching strategies when processing web pages" [7]. These differences are pronounced enough to allow for people with and without autism to be automatically differentiated between with $75 \%$ accuracy based on their gaze patterns alone [17]. At present, such differences are found mainly in longer and more highly variable eye-tracking scanpaths among web users with autism, indicating uncertainty in searching [6], and a focus on a larger amount of page elements irrelevant to the main task [7]. Notably, while there have been autismspecific efforts toward improving the accessibility of the text component $[18,16,19,15]$, investigations of the visual and organizational components of web pages are still very rare.

The available web accessibility guidelines for people with autism have not been evaluated, nor are based on empirical evidence of particular needs. For example, Britto and Pizzolato [3] collect and analyze a wide range of existing accessibility guidelines for people with autism from a total of nine countries and conclude that none of them are based on empirical research. The way the majority of these ASD-specific guidelines are obtained is through conducting literature reviews and matching the ASD diagnostic criteria to potential accessibility barriers $[12,8,4]$. This is also the case with the the Cognitive Accessibility User Research paper [13] issued by WC3, where the ASD-related recommendations are based on the ASD diagnostic criteria as a source of information for potential accessibility barriers and one interview with an anonymous user.

Our investigation into the accuracy and efficiency with which web users with autism process web pages is positioned within a currently existing gap between guidelines aiming to anticipate the needs of this population and a lack of empirical evidence as to what these needs actually are.

\section{DATA COLLECTION}

Overview: The stimuli used in our experiments were eight web pages. For each page, the participants were asked two inferential questions that required them to examine at least two visual elements to obtain the correct answer. While completing the task their eye movements were recorded. Participants had up to two minutes to answer the questions but if answered earlier could proceed to the next page. The questions were read out by the first author and answers were given verbally to minimise the impact of head movements and confounding factors (no use of mouse or keyboard required). The questions and answers had a very clear structure and are thus not an example of the type of social conversations that people with autism are known to struggle with (e.g. small talk, reading social cues, etc.).

Materials: To select the web pages, we took the top 100 websites listed by ALEXA.com and we considered their home pages, excluding those that were repeated more than once (e.g. we kept www.google.com but we removed www. google.co.uk). We then removed the pages that: i) were not in English, ii) were mainly designed for authentication, and iii) were mainly designed as search pages. We then used the VICRAM algorithm [14] to determine the complexity value of each web page. The complexity value (VCS) is between 0 and 10, hence, we introduced a threshold where: if VCS i 5 then "complex" otherwise "not complex". We also calculated the white space ratio (WSR) for the web pages based on the distance between the page elements The WSR is between 0 and 1 , hence: if WSR $i 0.5$ then "large space" otherwise "small space". As a result, our final selection of pages had a balanced representation of categories of pages with a high complexity (YouTube, Amazon, Adobe and BBC) and low complexity (WordPress, WhatsApp, Outlook and Netflix), as well as small (Outllok, Netflix, Adobe and BBC) and large space (WordPress, WhatsApp, YouTube and Amazon) between their elements.

Questions: The tasks were created following guidelines for exploratory search experiments [11]. They were designed to reflect the real-life use of these websites. There were two questions per web page (16 questions in total). Each question was designed in such a way that obtaining the right answer would require: i) visiting at least two web page elements, ii) using inference in order to use the information given on the web page to arrive to a third piece of information that was not explicitly presented. Examples of questions include: "What is the cheapest plan you can get that offers Email \& Live Chat support?" for the WordPress page, where the participant has to identify the plans that offer email and live chat support and compare their prices. Another example is "Which item has the largest price discount measured in percentage?" for the Amazon page. All questions spanned between 10 and 20 words (see repository).

Participants: A total of 41 adult participants took part in the study, of whom 19 had been formally diagnosed with autism. The inclusion criteria for the experimental group was a formal diagnosis of autism. All participants had to be over 18 years of age and to be able to use a computer. The exclusion criteria were any of the following: i) a diagnosis of any degree of intellectual disability, ii) a diagnosis of a reading disorder, and iii) conditions affecting vision that could not be corrected using glasses or lenses. For the control group, the inclusion and exclusion criteria were similar, except for having a diagnosis of autism. To ensure that no participants with a high incidence of autistic traits were included in the control group, all control participants completed the 50-item Autism Quotient (AQ) test [2]. This test is widely used as a screening tool for autism and usually represents the first step that general practitioners use toward providing the referral for a diagnosis by an expert. Of the 22 control group participants, one was excluded for having an "at risk" score $(>32)$ at the AQ test and two were excluded because of calibration issues. Thus, the retained data corresponds to 19 participants with autism (eight female and eleven male) and 19 control group participants (13 female and six male). All participants with ASD were recruited through a UK autism charity and through the student enabling center at the University of Wolverhampton. All control-group participants were recruited through snowball sampling. The mean age for the ASD group was $\mathrm{m}$ $=41.05$ with $\mathrm{SD}=14.04$, and $\mathrm{m}=32.15, \mathrm{SD}=9.93$ for the control group. 17 out of the 19 ASD participants answered a question about their education, where 11 people had completed a higher education degree and six people had a UK equivalent of a high-school degree (GCSE or A-levels). 15 people from the control group had completed a higher education degree and three people had completed A-levels 
Table 1: A between-group analysis of number of correct answers and response time (in seconds) [M: Mean, MD: Median, SD: Standard Deviation]

\begin{tabular}{|l|l|l|l|l|l|l|l|l|l|}
\hline \multirow{2}{*}{ Feature } & ASD Group & \multicolumn{3}{|c|}{ Control Group } & \multicolumn{3}{|c|}{ Mann-Whitney U } \\
\cline { 2 - 10 } & M & MD & SD & M & MD & SD & U & Z & p \\
\hline Answers & 0.91 & 1 & 0.28 & 0.93 & 1 & 0.25 & 54813 & -0.846 & 0.397 \\
\hline Response time & 30.42 & 28.62 & 12.44 & 23.54 & 21.44 & 9.31 & 7308 & -5.538 & $<\mathbf{0 . 0 0 0 1}^{* *}$ \\
\hline
\end{tabular}

Table 2: A comparative analysis between the ASD and control groups based on three features on all the pages for the synthesis tasks [ M: Mean, MD: Median, SD: Standard Deviation, NA: Not Applicable]

\begin{tabular}{|c|c|c|c|c|c|c|c|c|c|c|c|c|}
\hline \multirow[t]{2}{*}{ Feature } & \multicolumn{3}{|c|}{ ASD Group } & \multicolumn{3}{|c|}{ Control Group } & \multicolumn{3}{|c|}{ Independent $T$} & \multicolumn{3}{|c|}{ Mann-Whitney U } \\
\hline & $\mathbf{M}$ & MD & SD & $\mathbf{M}$ & MD & SD & $\mathrm{t}$ & df & $\mathbf{p}$ & $\mathbf{U}$ & $\mathbf{z}$ & p \\
\hline Mean Fixation Duration & 329.2 & 323 & 43.6 & 321.5 & 318.3 & 27.68 & NA & $\mathrm{NA}$ & NA & 150 & -0.89 & 0.191 \\
\hline Total Fixation Count & 84.86 & 78.5 & 19.8 & 66.4 & 63.25 & 13.05 & $\overline{\mathrm{NA}}$ & $\overline{\mathrm{NA}}$ & $\overline{\mathrm{NA}}$ & 89 & -2.67 & $0.004^{* *}$ \\
\hline N Transitions & 41.8 & 42.4 & 8.77 & 35.36 & 36 & 7.67 & 2.33 & 36 & $0.013^{*}$ & $\overline{\mathrm{NA}}$ & NA & NA \\
\hline
\end{tabular}

\begin{tabular}{|l|c|c|}
\hline & p Answers & p Response Time \\
\hline$\downarrow$ Complexity, $\uparrow$ Space & 0.6 & 0.063 \\
\hline$\downarrow$ Complexity, $\downarrow$ Space & 0.26 & $\mathbf{0 . 0 0 1}$ \\
\hline$\uparrow$ Complexity, $\uparrow$ Space & 0.95 & $\mathbf{0 . 0 0 8}^{*}$ \\
\hline$\uparrow$ Complexity, $\downarrow$ Space & 0.37 & $\mathbf{0 . 0 0 1}^{*}$ \\
\hline
\end{tabular}

Table 3: Between-group differences in answer correctness and response time per page type, where $\uparrow$ stands for High and $\downarrow$ stands for Low (MannWhitney $\mathrm{U}+$ Bonferroni correction of $\alpha=\mathbf{0 . 0 1 2 5})$

(equivalent to high school). All participants were highly independent adults, none of whom relied on a caregiver. The majority of the participants with ASD were either employed or enrolled in a higher education degree, while others (n $=6$ ) lived independently in council-provided housing and received disability benefits. The control-group participants were either employed or in education. All ASD participants were native speakers of English. 15 of the control group participants were native speakers of English and the remaining four had lived in the UK for many years and had a high level of English proficiency. All participants reported that they use the web on a daily basis, except for one ASD participant, who reported that she used it less than once a month.

Apparatus: The eye tracker used in the study was a Gazepoint GP3 video-based eye-tracker ${ }^{1}$ with a $60 \mathrm{~Hz}$ sampling rate and accuracy of 0.5-1 degree of visual angle. The screen shots of the web pages were presented on a 17 " LCD monitor. The distance between each participant and the eye-tracker was roughly $65 \mathrm{~cm}$. The gaze data was extracted and processed using the Gazepoint Analysis software.

\section{EXPERIMENTS}

The study implemented a between-group comparison design. Our dependent variables are accuracy and efficiency, measured through the following features:

- Answer correctness: This is a dichotomous variable indicating whether the answer given by each participant to each question was correct or incorrect. Answer correctness is directly related to accuracy, since an incorrect answer is indicative of a lower accuracy in obtaining the desired information and vice-versa.

- Answering time: The time in seconds taken by each participant to answer the questions for a given page.

\footnotetext{
${ }^{1}$ https://www.gazept.com/
}

This variable is related to efficiency, since taking a longer time to answer a question is indicative of a less efficient processing and vice-versa.

- Number of fixations: Gaze fixations refer to maintaining the visual gaze to a specific location on the screen [5]. The fixations reveal the scan paths of participants and their information-searching strategies.

- Average fixation duration: The average duration of fixations per participant per page measured in milliseconds. Longer fixations are considered to be related to higher cognitive effort when processing a task [5].

- Transitions between elements: This metric is informative of whether the gaze transitions between different page elements and how many.

The answer correctness variable is related to accuracy and the answering time variable is related to efficiency. We also compare answer correctness and response time for specific classes of pages based on complexity and space between elements. We do so in order to find out whether potential differences in accuracy and efficiency exist in all types of pages or were dominated by a particular type of pages. Furthermore, we analyse the eye-tracking metrics listed above, in order to gain a better understanding of the cause of any potential between-group differences. Considering the distribution of the dependent variable we use either the Independent T-test (parametric) or the Mann-Whitney U test (nonparametric).

\section{RESULTS AND DISCUSSION}

As can be seen from Table 1, the two groups did not have significant differences in their ability to answer the questions, however, the participants with ASD took significantly longer to do so. These results indicate that there were no differences in terms of accuracy as measured by the answers to the tasks; however, the users with ASD had a lower efficiency when completing the tasks. This effect remained stable when conducting the between-group analysis for particular classes of web pages (Table 3), where all p-values for answer correctness were above 0.05 and the the p-values for answering time showed significant differences for all page types except for low complexity and large space.

Looking for the root of these differences, the analysis of the gaze data (Table 2) shows that the longer times are not associated with longer fixations (no significant differences in mean fixation duration) but with a higher number of fixa- 
tions. Furthermore, these fixations are dispersed across a higher number of elements than those fixated by the control group, showing that the ASD participants have longer completion times associated with a higher number of fixations and a higher number of transitions between elements but with similar durations as the control group. The difference in number of transitions between elements suggests that the increased cognitive demand is potentially related to the way the elements are semantically organized into the visual space of the page. In addition, there is the possibility that the ASD group produced more fixations per element as they were occupied with investigating different aspects of an element before deciding on its role and relevance to the task. Should this be the case, a potentially useful future direction would be to investigate the homogeneity of individual page elements and whether having more homogeneous elements (e.g. containing only text or only images) would enhance efficiency. Finally, it is also possible that the ASD group experienced stronger priming effect from viewing the previously presented pages, however, the randomization of the stimuli prevents the analysis of specific patterns.

Our results with regards to accuracy should not be taken as conclusive, as it is possible that there was a ceiling effect related to the difficulty of the tasks and that more difficult tasks would result in some between-group differences. Rather, the value of these results is in showing that even relatively easy tasks elicit differences in the cognitive effort required to complete them successfully. Therefore, future web accessibility initiatives for people with high-functioning autism should focus on guidelines and interventions primarily aimed at reducing cognitive load. In addition, future accessibility studies for this population should always measure the trade-off between accomplishments and effort, as opposed to only showcasing the high information-processing ability of web users with high-functioning autism.

A natural next step for this study is to analyse the effects of complexity and space between elements, as well as the scanpaths of the participants. Further analysis of different eye-tracking metrics (e.g. the number of fixated elements not relevant to the task or the number of revisits for specific elements) would enable better understanding the design factors which potentially affect web users with autism. This analysis would allow us to provide a set of ASD-specific guidelines and/or empirical evaluation of existing guidelines.

\section{CONCLUSION}

We analysed the between-group differences in the accuracy and efficiency with which web users with and without autism completed information searching tasks on web pages. No differences in accuracy were found, but the ASD group took significantly longer to complete the tasks. Analysis of the eye-movement data allows the interpretation that the equal number of correct answers came with a higher price for the ASD group, as they had to put a higher cognitive effort to arrive at the same answers as the control group.

\section{REFERENCES}

[1] American Psychiatric Association. Diagnostic and Statistical Manual of Mental Disorders (5th ed.), 2013.

[2] S. Baron-Cohen, S. Wheelwright, R. Skinner, J. Martin, and E. Clubley. The autism-spectrum quotient (aq): Evidence from asperger syndrome/high-functioning autism, malesand females, scientists and mathematicians. Journal of autism and developmental disorders, 31(1):5-17, 2001.

[3] T. C. P. Britto and E. B. Pizzolato. Towards web accessibility guidelines of interaction and interface design for people with autism spectrum disorder. In proceedings of ACHI 2016 : The Ninth International Conference on Advances in Computer-Human Interactions, 2016.

[4] A. Darejeh and D. Singh. A review on user interface design principles to increase software usability for users with less computer literacy. Journal of Computer Science, 9(11):1443, 2013.

[5] A. T. Duchowski. Eye tracking methodology. Theory and practice, 328, 2007.

[6] S. Eraslan, V. Yaneva, Y. Yesilada, and S. Harper. Do web users with autism experience barriers when searching for information within web pages? In Proceedings of the 14th Web for All Conference on The Future of Accessible Work, page 20. ACM, 2017.

[7] S. Eraslan, V. Yaneva, Y. Yesilada, and S. Harper. Web users with autism: Eye tracking evidence for differences. Behaviour \&5 Information Technology, pages 1-23, 2018.

[8] M. G. Friedman and D. N. Bryen. Web accessibility design recommendations for people with cognitive disabilities. Technology and Disability, 19(4):205-212, 2007.

[9] U. Frith. Autism. Explaining the enigma. Blackwell Publishing, Oxford, UK, second edition, 2003.

[10] C. Laidi, J. Boisgontier, M. M. Chakravarty, S. Hotier, M.-A. dAlbis, J.-F. Mangin, G. A. Devenyi, R. Delorme, F. Bolognani, C. Czech, et al. Cerebellar anatomical alterations and attention to eyes in autism. Scientific reports, 7(1):12008, 2017.

[11] G. Marchionini. Exploratory search: From finding to understanding. Commun. ACM, 49(4):41-46, 2006.

[12] D. Moore. Computer-Based Learning Systems for People with Autism, chapter Disabled Students in Education: Technology, Transition, and Inclusivity, pages 84-108. IGI Global, 2011.

[13] L. Seeman and M. Cooper. Cognitive accessibility user research, jan 2015.

[14] O. Wu, W. Hu, and L. Shi. Measuring the visual complexities of web pages. ACM Transactions on the Web (TWEB), 7(1):1, 2013.

[15] V. Yaneva. Assessing text and web accessibility for people with autism spectrum disorder. $\mathrm{PhD}$ thesis, 2016.

[16] V. Yaneva and R. Evans. Six good predictors of autistic text comprehension. In Proceedings of the International Conference Recent Advances in Natural Language Processing, pages 697-706, 2015.

[17] V. Yaneva, L. A. Ha, S. Eraslan, Y. Yesilada, and R. Mitkov. Detecting autism based on eye-tracking data from web searching tasks. In Proceedings of the Internet of Accessible Things, page 16. ACM, 2018.

[18] V. Yaneva, I. P. Temnikova, and R. Mitkov. A corpus of text data and gaze fixations from autistic and non-autistic adults. In $L R E C, 2016$.

[19] V. Yaneva, I. P. Temnikova, and R. Mitkov. Evaluating the readability of text simplification output for readers with cognitive disabilities. In $L R E C, 2016$. 